\title{
On the Equivalence between KMS-States and Equilibrium States for Classical Systems
}

\author{
Michael Aizenman* \\ Department of Physics and Mathematics, Princeton University, Princeton, NJ, USA \\ Sheldon Goldstein $\star \star$ \\ Department of Mathematics, Cornell University, Ithaca, NY, USA \\ Christian Gruber \\ Laboratoire de Physique Théorique, CH-Lausanne, E.P.F., Switzerland \\ Joel L. Lebowitz ${ }^{\star \star \star}$ \\ Department of Physics, Belfer Graduate School of Sciences, New York, NY, USA \\ Philippe Martin \\ Laboratoire de Physique Théorique, CH-Lausanne, E.P.F., Switzerland
}

\begin{abstract}
It is shown that for any KMS-state of a classical system of noncoincident particles, the distribution functions are absolutely continuous with respect to Lebesgue measure; the equivalence between KMS states and Canonical Gibbs States is then established.
\end{abstract}

\section{Introduction}

In classical statistical mechanics macroscopic systems are described by "states" defined as probability measures on the phase space of the system. For infinite systems (the precise mathematical analogs of macroscopic systems) these measures are specified in terms of "local" distributions, i.e. $n$-particle distributions on bounded regions $\Lambda \subset \mathbb{R}^{v}, \mu_{\Lambda}^{(n)}[1]$. Moreover, systems at equilibrium are specified by states satisfying certain conditions (e.g. D.L.R. equations $\equiv$ Gibbs states, K.-S.equations, limit of finite volume grand-canonical states, variational principles). These conditions have been proved to be equivalent in many different cases [2-5]; however, in these proofs, it has always been assumed that the $\mu_{A}^{(n)}$ are absolutely continuous with respect to Lebesgue measure. This assumption gives thus a special status to the Lebesgue measure which has not been derived from physical principles.

On the other hand, it has been suggested that equilibrium states could also be defined as states satisfying the KMS-condition; in fact it is well known that for infinite quantum systems, Gibbs states are characterized by this KMS condition [6]; for infinite classical systems, Gallavotti and Verboven gave in a recent work [7] some sufficient conditions for the equivalence of the KMS-conditions and the Kirkwood-Salzburg equations; among those fairly strong conditions were in particular the conditions of absolute continuity of the $\mu_{\Lambda}^{(n)}$ with respect to Lebesgue measure, low density, exponential clustering, smoothness properties, ....

* $\quad$ Supported in part by NSF Grant MCS 75-21684

$\star \star$ Supported in part by NSF Grant MPS 72-04534

$\star \star \star$ Supported in part by NSF Grant MPS 75-20638 
In this paper, we shall deduce the (Canonical) Gibbs states directly from the KMS-condition without any prior assumption about the absolute continuity of the $\mu_{\Lambda}^{(n)}$. Our result is valid for a very large class of interactions and for all densities (below close packing in the case of hard cores). The physical significance of the classical KMS-condition lies in the fact that it yields a definition of equilibrium states which is related to the dynamics of the system rather than to the second principle of equilibrium thermodynamics; indeed it was shown $[8,9]$ that the KMS-condition is a fairly direct consequence of a stability condition of the state under local perturbation of the Hamiltonian time evolution; moreover it has also been shown that Gibbs states are invariant under the time evolution [10]; this is obviously the minimal requirement for a state to describe a system in equilibrium. The converse statement, "any time invariant state is a Gibbs state", is certainly not true generally without further assumptions. What we do then in this paper is to show that the requirement of time invariance plus "stability" (plus unfortunately some ergodic properties which can however be relaxed, [11]), which lead to the KMS-condition, does indeed single out the Gibbs states as the proper description of equilibrium systems.

In Section 2, we recall the mathematical description of infinite classical systems and the definition of the "static KMS condition"; in Section 3, we prove that any KMS-state yields distributions which are absolutely continuous with respect to Lebesgue measure and that KMS-states are canonical Gibbs states. It follows then from recent work of Georgi [12] and others [13] that any KMS-states is a superposition of extremal Gibbs states with temperature $T$ and different activities.

\section{Mathematical Description of Classical Systems}

1) A classical system of particles with hard core of diameter $d \geqq 0$, moving in a bounded region $\Lambda$ of the physical space $\mathbb{R}^{v}$, is described by the phase space $\Omega_{\Lambda}$ of finite subsets of $\Lambda \times \mathbb{R}^{v}$ satisfying the hard core condition, $[1,14]$ i.e.:

$$
\begin{aligned}
& \Omega_{\Lambda, n}=\left\{X^{n}=\left\{x_{1}, \ldots, x_{n}\right\} ; x_{i}=\left(q_{i}, p_{i}\right) \in \Lambda \times \mathbb{R}^{v}\right. \\
& \left.\cdot\left|q_{i}-q_{j}\right|>d \text { for all } i \neq j\right\} \\
& \Omega_{\Lambda}=\bigcup_{n=0}^{\infty} \Omega_{\Lambda, n} .
\end{aligned}
$$

On the other hand, an infinite classical system is described by the phase space $\Omega$ of infinite countable subsets of $\mathbb{R}^{v} \times \mathbb{R}^{v}$ satisfying the hard core condition and the condition that the number of particles in any bounded region $\Lambda$ of $\mathbb{R}^{v}$ is finite. Introducing with any Borel set $\Lambda$ in $\mathbb{R}^{v}$ the projection $\Pi_{\Lambda}: X \mapsto X_{\Lambda}$ of $\Omega$ into $\Omega_{\Lambda}$ where

$$
X_{\Lambda}=\left\{x_{i} ; x_{i}=\left(q_{i}, p_{i}\right) \in X, q_{i} \in \Lambda\right\}
$$

we have:

$$
\Omega=\left\{X=\left\{x_{i}\right\} ; x_{i} \in \mathbb{R}^{v} \times \mathbb{R}^{v},\left|q_{i}-q_{j}\right|>d \text { for all } i \neq j^{1},\right.
$$

for any bounded $\Lambda$ the set $X_{\Lambda}$ is finite $\}$.

1 Notice that even for $d=0, X \in \Omega$ does not contain coincident particles-two or more particles with the same position and momentum 
Moreover, for any Borel subset $\Lambda$ of $\mathbb{R}^{v}$, and $\Lambda^{c}=\mathbb{R}^{v} / \Lambda$, we have:

$\Omega \tilde{\subset} \Omega_{\Lambda} \times \Omega_{\Lambda^{c}}$ with $\Omega \cong \Omega_{\Lambda} \times \Omega_{\Lambda^{c}}$ if $d=0$.

In the following we shall denote by $X$ the configurations in $\Omega_{\Lambda}$ and by $Y$ the configurations in $\Omega_{\Lambda^{c}}$; we shall say that $X \in \Omega_{\Lambda}$ and $Y \in \Omega_{\Lambda^{c}}$ are compatible if $X \cup Y \in \Omega$.

2) The classical system is furthermore characterized by a potential $\Phi$, function on the (non empty) finite subsets of the physical space $\mathbb{R}^{v}$; alternatively $\Phi$ can be considered as a symmetric function defined on $\bigcup_{n \geqq 1} \mathbb{R}^{v n}$

$$
\Phi\left(\left\{q_{1}, \ldots, q_{n}\right\}\right)=\Phi\left(q_{1}, \ldots, q_{n}\right) .
$$

For any bounded domain $\Lambda \subset \mathbb{R}^{v}$ and any $Y \in: \Omega_{\Lambda^{c}}$ the Hamiltonian $H_{A, Y}$ of the finite system $\Lambda$ with boundary condition $Y$ is the function on $\Omega_{\Lambda}$ defined formally by:

$$
H_{\Lambda, Y}(X)=\sum_{x_{i}=\left(q_{i}, p_{i}\right) \in X} \frac{p_{i}^{2}}{2 m}+\sum_{\substack{\phi \overline{\bar{X}} \subset X \\ \bar{Y} \subset Y}} \Phi(\bar{X} \cup \bar{Y}) .
$$

3) The states are defined as probability measure $\mu$ on the phase space; for finite systems the measurable space $\left(\Omega_{A}, \mathscr{B}_{A}\right)$ is defined by the $\sigma$-algebra $\mathscr{B}_{\Lambda}$ of subsets of $\Omega_{\Lambda}$ introduced in the following manner [10]:

i) $\mathscr{B}_{\Lambda, n}$ is the $\sigma$-algebra of subsets of $\Omega_{\Lambda, n}$ isomorphic to the $\sigma$-algebra of symmetric Borel subsets of $\left(\Lambda \times \mathbb{R}^{v}\right)_{d}^{n}$, where:

$$
\left(\Lambda \times \mathbb{R}^{v}\right)_{d}^{n}=\left\{\underline{X}^{n}=\left(x_{1}, \ldots, x_{n}\right) ; x_{i} \in \Lambda \times \mathbb{R}^{v},\left|q_{i}-q_{j}\right|>d\right\} .
$$

ii) $\mathscr{B}_{\Lambda}=\left\{A \subset \Omega_{\Lambda} ; A \cap \Omega_{\Lambda, n} \in \mathscr{B}_{\Lambda, n}\right\}$.

For infinite systems, the measurable space $(\Omega, \mathfrak{B})$ is defined using the $\mathscr{B}_{\Lambda}, \Lambda$ bounded Borel set in $\mathbb{R}^{v}$, as cylinder sets; i.e. $\mathfrak{B}$ is the $\sigma$-algebra of subsets of $\Omega$ generated by $\Pi_{\Lambda}^{-1} A, A \in \mathscr{B}_{\Lambda}$. In fact $\mathfrak{B}$ is obtained by means of the weakest topology making the function $\sum_{x_{i} \in X} h\left(x_{i}\right)$ continuous for all continuous functions $h$ with compact support in $q$.

Remarks. a) Since $\Omega_{\Lambda, n}$ is isomorphic to the symmetrization of $\left(\Lambda \times \mathbb{R}^{v}\right)_{d}^{n}$, any function on $\Omega_{\Lambda, n}$ can be considered as a symmetric function on $\left(\Lambda \times \mathbb{R}^{v}\right)_{d}^{n}$ and we shall use the same notation for those two functions.

b) In the same manner, any Borel measure $\mu$ on $\left(\Omega_{\Lambda, n}, \mathscr{B}_{\Lambda, n}\right)$ can be considered as a symmetric Borel measure on $\left(\Lambda \times \mathbb{R}^{v}\right)_{d}^{n}$ which we shall also denote by $\mu$ such that

$$
\int_{\Omega_{\Lambda, n}} d \mu \cdot f=\int_{\left(\Lambda \times \mathbb{R}^{v}\right)_{d}^{n}} d \mu\left(x_{1}, \ldots, x_{n}\right) f\left(x_{1}, \ldots, x_{n}\right) .
$$

The measure $\mu$ has furthermore the following property which we shall need to deal with the symmetry condition imposed on the functions (see Prop. 2 of Sec. 3). Let $D_{\alpha}^{\pi}$, $\alpha \in \mathbb{R}^{v}, \pi$ a permutation of $(1,2, \ldots n)$, be the open ordered subset of $\left(\Lambda \times \mathbb{R}^{v}\right)_{d}^{n}$ defined by

$$
D_{\alpha}^{\pi}=\left\{\left(x_{1}, \ldots, x_{n}\right) \in\left(\Lambda \times \mathbb{R}^{v}\right)_{d}^{n} ; q_{\pi_{1}} \cdot \alpha<q_{\pi_{2}} \cdot \alpha<\ldots<q_{\pi_{n}} \cdot \alpha\right\}
$$


and $g$ be any bounded function with support in $D_{\alpha}^{\pi}$; then, since the permuted domains are disjoint, we have:

$$
\int_{\Omega_{\Lambda, n}} d \mu g_{\mathrm{sym}}=\int_{(\Lambda \times \mathbb{R} v)_{d}^{n}} d \mu\left(x_{1}, \ldots, x_{n}\right) g\left(\alpha_{1}, \ldots, a_{n}\right)
$$

with

$$
g_{\mathrm{sym}}\left(\left\{x_{1}, \ldots, x_{n}\right\}\right)=\frac{1}{n !} \sum_{\pi} g\left(x_{\pi_{1}}, \ldots, x_{\pi_{n}}\right) .
$$

4) Since the space $(\Omega, \mathfrak{B})$ without hard core is a polish space under the natural topology, our phase space is a Borel subset of a polish space and thus for any state $\mu$ there exists conditional probability measures $\mu_{\Lambda}[d X ; Y]$ defined for any bounded measurable domain $\Lambda \subset \mathbb{R}^{v}$ and $Y \in \Omega_{\Lambda^{c}}$ as a probability measure on $\Omega_{\Lambda}$ such that:

i) $\mu_{\Lambda}[d X ; Y]=\chi_{d}(X \cup Y) \mu_{\Lambda}[d X ; Y]$, where $\chi_{d}$ is the characteristic function of $\Omega$ in $\Omega_{\Lambda} \times \Omega_{\Lambda^{c}}$;

ii) for any measurable set $\Delta$ in $\mathscr{B}_{\Lambda}$ the mapping $Y \mapsto \mu_{\Lambda}[\Delta ; Y]$ of $\Omega_{\Lambda^{c}}$ in $\mathbb{R}^{v}$ is measurable;

iii) for any bounded measurable function $f$ on $\Omega$

$$
\int_{\Omega} d \mu \cdot f=\int_{\Omega_{\Lambda^{c}}} \mu_{\Lambda^{c}}[d Y] \int_{\Lambda_{\Lambda}} \mu_{\Lambda}[d X ; Y] f(X \cup Y),
$$

where for any Borel set $M$ in $\mathbb{R}^{v}, \mu_{M}$ is the Borel measure on $\Omega_{M}$ induced by the projection $\Pi_{M}$.

iv) Compatibility Condition: Let $\Lambda$ and $M$ be bounded Borel domains in $\mathbb{R}^{v}$ such that $M \supset \Lambda$ and let us denote by $X, T, Y$ respectively configurations in $\Omega_{\Lambda}, \Omega_{M / \Lambda}$, $\Omega_{M^{c}}$; then the conditional probabilities satisfy for almost all $Y$ (w.r. to $\mu_{M^{c}}$ ) the equation

$$
\mu_{M}[d X d T ; Y]=\mu_{\Lambda}[d X ; T \cup Y] \int_{\Omega_{A}} \mu_{M}[d \bar{X} d T ; Y]
$$

5) A state $\mu$ is called a Gibbs State for the interaction $\Phi$ with inverse temperature $\beta$ and fugacity $z$, if for every bounded open $\Lambda \subset \mathbb{R}^{v}$ the conditional probabilities satisfy the D.L.R. equations:

$$
\mu_{\Lambda}[d X ; Y]=\chi_{d}(X \cup Y) \frac{e^{-\beta H_{\Lambda, Y}(X)}}{Q(\Lambda, Y)} z^{|X|} d X,
$$

where

$$
d X \cong \sum_{n=0}^{\infty} \frac{1}{\bar{n}} d x_{1} \ldots d x_{n}, \quad|X|=\text { cardinality of the set } X
$$

and

$$
Q(\Lambda, Y)=\int_{\Omega_{\Lambda}} d X \chi_{d}(X \cup Y) e^{-\beta H_{\Lambda, Y}(X)} z^{|X|} .
$$

On the other hand a state $\mu$ is called [12] a "Canonical Gibbs State for the interaction $\Phi$ with inverse temperature $\beta$ ", if for every bounded open $\Lambda \subset \mathbb{R}^{v}$, the 
conditional probabilities satisfy for almost all $Y$ in $\Omega_{A^{c}}$ the Canonical Equation (C.E.)

$$
\mu_{\Lambda}\left[d X^{n} ; Y\right] \cong \chi_{d}\left(X^{n} \cup Y\right) e^{-\beta H_{A, Y}\left(X^{n}\right)} C_{A, Y, n} d x_{1} \ldots d x_{n}
$$

with $C_{A, Y, n}$ non negative constant.

The classical KMS-states are defined with respect to a suitable class $\mathfrak{A}$ of functions on the phase space; this class $\mathfrak{A}$ is however not unique and we shall want to choose $\mathfrak{Q I}$ as small as possible; our choice of $\mathfrak{Q H}$ will be guided by the following considerations.

In the description of states in terms of correlation measures $d \varrho$ [7] one introduces the algebra $\tilde{\mathscr{I}}$ of real functions $f$ on $\Omega$ given by

$$
f(X)=\sum_{n \geqq 0} \sum_{\bar{X}^{n} \subset X} f_{T}^{n}\left(\bar{X}^{n}\right) \quad \text { or simply } f=S f_{T},
$$

where $f_{T}^{n}$ are $C^{\infty}$-functions on $\left(\mathbb{R}^{v} \times \mathbb{R}^{v}\right)^{n}$ which are symmetric, have compact support and are identically zero, except for finitely many values of $n$. The interest of this algebra $\tilde{\mathscr{A}}$, also called "algebra of unbounded strictly local observables", lies in the definition of correlation measures $\varrho$, which give for integrable $f$ in $\tilde{\mathfrak{U}}$

$$
\mu[f]=\int_{\Omega} d \mu f=\sum_{n \geqq 0} \int_{\Omega_{n}} d \varrho\left(X^{n}\right) f_{T}^{n}\left(X^{n}\right) .
$$

On the other hand in the description of states in terms of probability measures $\mu$, one introduces rather the algebra of bounded strictly local observables [1].

For our purpose a convenient class $\mathfrak{A}$ will be given by the algebra generated by the functions $e^{i S h} \cdot f$ with $f \in \tilde{\mathscr{A}}$ and $h \in \mathscr{D}=C^{\infty}$-real functions on $\mathbb{R}^{v} \times \mathbb{R}^{v}$ with compact support in the $q$ variable. In fact $\mathscr{U}$ is only a slight enlargement of $\tilde{\mathscr{A}}$ and the usefulness of the functions

$$
W_{h}=\exp i S h
$$

will be seen in the proofs of next sections.

Definition. A state $\mu$ of the classical system is called a (static) KMS-state for the interaction $\Phi$ and inverse temperature $\beta$ if

1) $\mathscr{R} \subset \mathscr{L}^{1}[\mu]$,

2) $g \cdot\{f, H\} \in \mathscr{L}^{1}[\mu]$ for all $f, g$ in $\mathscr{Q}$,

3) $\mu[\{f, g\}]=\beta \mu[g \cdot\{f, H\}]$ for all $f, g$ in $\mathscr{R}$,

where the Poisson bracket $\{f, g\}$ is the bilinear map of $\mathscr{A} \times \mathfrak{H} \rightarrow \mathscr{U}$ defined by $\{f, g\}$ $=\sum_{i} \frac{\partial f}{\partial q_{i}} \frac{\partial g}{\partial p_{i}}-\frac{\partial f}{\partial p_{i}} \frac{\partial g}{\partial q_{i}}$

and

$$
\{f, H\}(X)=\sum_{x=(q, p) \in X}\left[\frac{\partial f(x)}{\partial q} \cdot \frac{p}{m}-\frac{\partial f(x)}{\partial p} \sum_{\substack{X \subset X \\ X \ni x}} \frac{\partial \Phi(\bar{X})}{\partial q}\right] .
$$

Let us remark that 1) expresses in particular the condition that the expected number of particles in any finite region is finite. The condition 2) will be satisfied in 
particular for potential $\Phi$ which are $C^{\infty}$-functions with finite range; however, it also allows functions of class $C^{1}$ which are singular on the boundary of $\left(\Lambda \times \mathbb{R}^{v}\right)_{d}^{n}$. Let us also note that for all $h \in \mathscr{D}$ and $f \in \mathfrak{U}$

$$
\left\{W_{h}, f\right\}=i W_{h}\{S h, f\} \text {. }
$$

\section{Equivalence of KMS-States with Canonical Gibbs States}

In this section we shall establish our main theorem, which then will yield the equivalence between KMS-states with canonical Gibbs states. To express this theorem in a form which is valid for systems with hard core $d \neq 0$ we introduce the following definition.

Definition. A state $\mu$ is "loosely packed" if for every bounded connected $\Lambda$ in $\mathbb{R}^{v}$ and almost all $Y$ in $\Omega_{A^{c}}$, there exists a bounded $M \supset \Lambda$ such that for any pairs of configurations $X, X^{\prime}$ in $\Omega_{\Lambda, n}$ compatible with $Y$, the points $X \cup Y_{M}$ and $X^{\prime} \cup Y_{M}$ belong to a connected subset of $\Omega_{M, n+\left|Y_{M}\right|}$.

Roughly speaking this condition requires that for almost all configurations in $\Omega$ the "free volume" is sufficiently large; for translation invariant state this condition will be satisfied whenever the density is smaller then the close packing density.

In the rest of this paper, we shall always denote by $\Lambda$ a bounded open set in $\mathbb{R}^{v}$; moreover, we shall assume that the potential $\Phi$ is given by functions $\Phi^{n}\left(q_{1}, \ldots, q_{n}\right)$ on $\mathbb{R}^{v n}$ which are symmetric, of class $\mathscr{C}^{1}$ on $\left(\mathbb{R}^{v}\right)_{d}^{n}$, and such that for all $X \in \Omega, x \in X$,

$$
\sum_{x \in \bar{X} \subset X}|\Phi(\bar{X})|<\infty^{2}
$$

and

$$
\sum_{(q, p)=x \in \bar{X} \subset X} \frac{|\partial \Phi(\bar{X})|}{\partial q}<\infty \text {, uniformly in a neighborhood of } q .
$$

Theorem 1. Let $\mu$ be a loosely packed KMS-state; then the conditional probability measures are absolutely continuous with respect to Lebesgue measure and $\mu$ is a canonical Gibbs state.

To prove this theorem, we shall need the following two lemmas; the first lemma reduces the problem to finite systems with exactly $n$ particles, and the second proves the equivalence of KMS states with canonical Gibbs states for such finite systems.

Lemma 1. For any KMS-state $\mu$ of the infinite system, the conditional probability $\mu_{\bar{\Lambda}}[d X ; Y]$ with $\bar{\Lambda}$ the closure of $\Lambda$ satisfies for almost all $Y$ the following "local" KMS-condition

$$
\int_{\Omega_{\bar{\Lambda}, n}} \mu_{\bar{\Lambda}}\left[d X^{n} ; Y\right] \cdot\left[\{f, g\}-\beta g\left\{f, H_{\bar{\Lambda}, Y}\right\}\right]\left(X^{n}\right)=0
$$

for all symmetric functions $f, g$ of class $\mathscr{C}^{\infty}$ with compact support contained in $\left(\Lambda \times \mathbb{R}^{v}\right)^{n}$.

For $d=0$ these conditions are much too strong. In particular, the first inequality implies in this case that $\Phi$ has finite range. It might be better, for $d=0$, to include in the definition of a KMS state $\mu$ that (14) holds for $\mu$, a.e. $X \in \Omega$ 
Lemma 2. Let $\mu$ be any KMS-state of the finite system $\Omega_{\bar{\Lambda}, n}$ for the Hamiltonian $H=$ $\sum_{i=1}^{n} \frac{p_{i}^{2}}{2 m}+U\left(q_{1}, \ldots, q_{n}\right)$ with $U$ of the class $\mathscr{C}^{1}$ on $\left(\Lambda \times \mathbb{R}^{v}\right)_{d}^{n}$; then $\mu$ is absolutely continuous with respect to Lebesgue measure on each connected open subset $\mathscr{C}$ of $\left(\Lambda \times \mathbb{R}^{v}\right)_{d}^{n}$ and is given on $\mathscr{C}$ by:

$$
d \mu=C \exp \left[-\beta H\left(X^{n}\right)\right] d x_{1} \ldots d x_{n}
$$

with $C$ a positive real number.

Let us remark that in these lemmas we have used $\bar{\Lambda}$ (i.e. $\bar{\Lambda}^{c}$ is open) in view of the application of Property 1 below which is valid for open set. We shall show at the end of the proof of Theorem 1 that both of these lemmas also hold with $\bar{\Lambda}$ replaced by $\Lambda$.

Proof of Lemma 1. With any Borel set $M$ in $\mathbb{R}^{v}$, let $\mathfrak{A}_{M}$ be the subalgebra of $\mathfrak{A}$ generated by the functions $W_{h} \cdot f$ for which support $h \subset M \times \mathbb{R}^{v}$ and support $f_{T}^{n} \subset\left(M \times \mathbb{R}^{v}\right)^{n}$. We note that:

$$
\{f, g\}=0 \text { for } f \in \mathfrak{A}_{M} \text { and } g \in \mathfrak{A}_{N} \text { if } M \text { and } N
$$

are disjoint.

From the KMS-condition, it follows that for any bounded open $\Lambda$ in $\mathbb{R}^{v}$ and any $f, g \in \mathfrak{A}_{\Lambda}, g_{c} \in \mathfrak{A}_{\bar{\Lambda}^{c}}$, we have

$$
\mu\left[g_{c}\{f, g\}\right]=\beta \mu\left[g_{c} g\{f, H\}\right]
$$

which yields

$$
\int_{\Omega_{\bar{\Lambda}^{c}}} \mu_{\bar{\Lambda}^{c}}[d Y] g_{c}(Y) F(Y)=0 \text { for all } g_{c} \in \mathfrak{A}_{\bar{\Lambda}^{c}}
$$

with

$$
F(Y)=\int_{\Omega_{\bar{A}}} \mu_{\bar{\Lambda}}[d X ; Y]\left[\{f, g\}-\beta g\left\{f, H_{\bar{\Lambda}, Y}\right\}\right](X) .
$$

To conclude that $F(Y)=0$ a.e. we first remark that $F$ and $g_{c} F$ are in $\mathscr{L}^{1}\left[\mu_{\bar{\Lambda}^{c}}\right]$ since $\left\{f, g g_{c}\right\}$ and $g g_{c}\{f, H\}$ are in $\mathscr{L}^{1}[\mu]$; we then need the following property:

Property 1. Let $M$ be any open set in $\mathbb{R}^{v}$ and let $\mu^{1}, \mu^{2}$ be two positive finite measures on $\Omega_{M}$ such that $\mathfrak{A}_{M} \subset \mathscr{L}^{1}\left[\mu^{1}\right] \cap \mathscr{L}^{1}\left[\mu^{2}\right]$ and $\mu^{1}[g]=\mu^{2}[g]$ for all $g$ in $\mathfrak{A}_{M}$; then $\mu^{1}$ $=\mu^{2}$ (i.e. $\mathfrak{A}_{M}$ is separating for finite measures on $\Omega_{M}$ ).

To establish this Property, let $\mathcal{O}$ be a bounded open set in $M$, and $h^{\alpha}(q)$ be a sequence of $C^{\infty}$-functions with support in $\mathcal{O}$ converging pointwise to $\theta \chi_{\mathcal{O}}$ where $\theta \in \mathbb{R}$ and $\chi_{\mathcal{O}}$ is the characteristic function of $\mathcal{O}$. For any $g$ in $\mathfrak{U}_{\mathscr{O}}$, the functions $W_{h^{\alpha}} \cdot g$ are in $\mathfrak{A}_{\mathscr{O}}$ and converge pointwise to $W_{\theta \chi \mathfrak{O}} \cdot g$; moreover by dominated convergence, we have

$$
\lim _{\alpha \rightarrow \infty}\left(\mu^{1}-\mu^{2}\right)\left[W_{h^{\alpha}} \cdot g\right]=\sum_{k=0}^{\infty} e^{i k \theta} \int_{\Omega_{\mathcal{O}, k}}\left[d \mu_{\mathscr{O}}^{1}\left(Y^{k}\right)-d \mu_{\mathscr{O}}^{2}\left(Y^{k}\right)\right] g\left(Y^{k}\right)=0
$$

for all $\theta \in \mathbb{R}$.

Since $g \in \mathscr{L}^{1}\left[\mu^{1}\right] \cap \mathscr{L}^{1}\left[\mu^{2}\right]$, the coefficient of this trigonometric series are summable and hence they must all vanish; choosing $g=S g_{T}$ with $g_{T}^{k} \neq 0$ only for $k$ 
$=n$, we conclude that for any bounded open set $\mathcal{O}$ in $M$ and any $n$

$$
\int_{\Omega \mathscr{O}, k}\left[d \mu_{\mathscr{O}}^{1}\left(Y^{n}\right)-d \mu_{\mathscr{O}}^{2}\left(Y^{n}\right)\right] g_{T}^{n}\left(Y^{n}\right)=0
$$

for all functions $g_{T}^{n}\left(y_{1}, \ldots, y_{n}\right)$ of class $C^{\infty}$ with compact support in $\left(\mathcal{O} \times \mathbb{R}^{v}\right)^{n}$. It thus follows that $\mu^{1}=\mu^{2}$.

To apply this Property to our case, we decompose $F$ into

$$
F(Y)=F_{+}(Y)-F_{-}(Y) \quad F_{ \pm}(Y) \geqq 0
$$

and set $\mu^{1}=\mu_{\bar{\Lambda}^{c}} F_{+}, \mu^{2}=\mu_{\bar{\Lambda}^{c}} F_{-}$. Since $F$ and $g_{c} F$ are in $\mathscr{L}^{1}\left[\mu_{\bar{\lambda}^{c}}\right]$, the measures $\mu^{1}, \mu^{2}$ satisfy the conditions of Property 1 with $M=\bar{\Lambda}^{c}$; we thus have

$$
\mu_{\bar{\Lambda}^{c}}[d Y] F_{+}(Y)=\mu_{\bar{\Lambda}^{c}}[d Y] F_{-}(Y)
$$

and thus

$$
F(Y)=\sum_{n=0}^{\infty} \int_{\Omega_{\bar{\Lambda}, n}} \mu_{\bar{\Lambda}}\left[d X^{n} ; Y\right]\left[\{f, g\}-\beta g\left\{f, H_{\bar{\Lambda}, Y}\right\}\right]\left(X^{n}\right)=0
$$

$\mu_{\bar{\Lambda}^{c}}$ almost everywhere.

To conclude the proof of Lemma 1, we still need to show that each term of the above sum is zero. We first remark that Equation (15) remains valid with $F(Y)$ replaced by $F_{\theta}(Y)$ where

$$
F_{\theta}(Y)=\sum_{k=0}^{\infty} e^{i k \theta} \int_{\Omega_{\bar{A}, k}} \mu_{\bar{\Lambda}}\left[d X^{k} ; Y\right]\left[\{f, g\}-\beta g\left\{f, H_{\bar{\Lambda}, Y}\right\}\right]\left(X^{k}\right) .
$$

To establish this result let $M$ be any closed set in $\bar{\Lambda}^{c}, f=S f_{T}, g=S g_{T}$ be any functions in $\mathfrak{A}_{\Lambda}$ and $g_{c} \in \mathfrak{A}_{M}$; moreover let $h^{\alpha}$ be a sequence of real functions in $\mathscr{D}$ such that $h^{\alpha}(q)=\theta$ on $\Lambda$ and $h^{\alpha}(q)=0$ on $M$, converging pointwise to $\theta \chi_{\bar{\Lambda}}$. Since $\left\{f, g_{c}\right\}$ $=0$ from the above remark, $\left\{f, W_{h^{\alpha}}\right\}=0$ since $h_{\alpha}$ is constant on the support of $f_{T}$, and $g g_{c} h_{\alpha}$ belongs to $\mathfrak{A}$, we obtain from the KMS-condition

$$
\mu\left[g_{c} W_{h^{\alpha}}\{f, g\}\right]=\beta \mu\left[g_{c} W_{h^{\alpha}} g\{f, H\}\right]
$$

and Equation (15) with $F$ replaced by $F_{\theta}$ follows by dominated convergence.

Repeating the above argument we conclude that $F_{\theta}(Y)=0$ for almost all $Y$ and there exists a set of full $\mu_{\bar{\Lambda}^{c}}$-measure such that $F_{\theta}(Y)=0$ for all $\theta \in \mathbb{R}^{3}$. Again the coefficient of the trigonometric series Equation (16) are summable and hence vanish. Choosing $f$ and $g$ generated by $n$-point functions $f_{T}^{n}, g_{T}^{n}$, we obtain

$$
\int_{\Omega \bar{A}, n} \mu_{\bar{\Lambda}}\left[d X^{n} ; Y\right]\left[\left\{f_{T}^{n}, g_{T}^{n}\right\}-\beta g_{T}^{n}\left\{f_{T}^{n}, H_{\bar{A}, Y}\right\}\right]\left(X^{n}\right)=0
$$

for almost all $Y$.

By separability of the algebra of $\mathscr{C}^{\infty}$-functions, this equation then holds on a common set of full $\mu_{\bar{\Lambda}^{c}}$-measure for all $f^{n}, g^{n}$ of class $\mathscr{C}^{\infty}$ with compact support in $\left(\Lambda \times \mathbb{R}^{v}\right)^{n}$, which concludes the proof of Lemma 1 .

3 Remove a countable union of null sets corresponding to rational $\theta$ 's; then $F_{\theta}(Y)=0$ for all real $\theta$ by continuity 
Proof of Lemma 2. 1) $\mu$ satisfies the KMS-condition

$$
\mu[\{f, g\}]=\beta \mu[g\{f, H\}]
$$

for any symmetric $\mathscr{C}^{\infty}, f, g$ with compact support in $\left(\Lambda \times \mathbb{R}^{v}\right)^{n}$. Using the identity

$$
\left\{f, g e^{-\beta H}\right\}=e^{-\beta H}[\{f, g\}-\beta g\{f, H\}]
$$

and the property of the potential $U$, it follows that

$$
\bar{\mu}\left[\left\{f, g e^{-\beta H}\right\}\right]=0
$$

for all $\mathscr{C}^{\infty}, f, g$ with support in $\left(\Lambda \times \mathbb{R}^{v}\right)_{d}^{n}$, where $\bar{\mu}$ is the positive Borel measure on $\Omega_{\bar{\Lambda}, n}$ defined by

$$
\bar{\mu}=e^{\beta H} \mu \text {. }
$$

Moreover, by density, Equation (18) holds also for $g$ 's of class $\mathscr{C}^{1}$ and thus

$$
\bar{\mu}[\{f, g\}]=0
$$

is valid of all $\mathscr{C}^{1}-f, g$ with support in $\left(\Lambda \times \mathbb{R}^{v}\right)_{d}^{n}$. With the remark of Section $2 \bar{\mu}$ yields a symmetric measure on $\left(\Lambda \times \mathbb{R}^{v}\right)_{d}^{n}$ which has the property

$$
\int_{\left(\Lambda \times \mathbb{R}^{v}\right)_{d}^{n}} d \bar{\mu}\left(x_{1}, \ldots, x_{n}\right) \quad\{f, g\}\left(x_{1}, \ldots, x_{n}\right)=0
$$

for all $f, g$ of class $\mathscr{C}^{1}$ with compact support in some open ordered set $D_{\alpha}^{\pi}$ of $\left(\Lambda \times \mathbb{R}^{v}\right)_{d}^{n}$.

2) To conclude the proof, we have to show that $\bar{\mu}$ is absolutely continuous with respect to Lebesgue measure on $\mathscr{C}$, where $\mathscr{C}$ is an open connected domain of $\left(\Lambda \times \mathbb{R}^{v}\right)_{d}^{n}$. For this, we note that it is always possible to decompose $\mathscr{C}$ as a union of connected open subsets of $D_{\alpha}^{\pi}$, defined by Equation (5)

$$
\mathscr{C}=\bigcup_{\pi, \alpha} \mathscr{C} \cap D_{\alpha}^{\pi}
$$

Then absolute continuity on $\mathscr{C}$ will follow if we have absolute continuity on each of the $D_{\alpha}^{\pi}$ s. For this latter fact, we need the property:

Property 2. Let $\bar{\mu}$ be any positive Borel measure on $\left(\Lambda \times \mathbb{R}^{v}\right)_{d}^{n}$ and $\mathcal{O}$ be any connected open set in $\left(\Lambda \times \mathbb{R}^{v}\right)_{d}^{n}$ such that:

$$
\int d \bar{\mu}\{f, g\}=0 \quad \text { for all (not necessarily symmetric) }
$$

$f, g$ of class $\mathscr{C}^{1}$ with compact support in $\mathcal{O}$; then $d \bar{\mu}=C d x_{1} \ldots d x_{n}$ on $\mathcal{O}, c>0$.

Proof. Let $h\left(x_{1}, \ldots, x_{n}\right)$ be a function in $\mathscr{C}_{\mathscr{O}}^{1}$ which equals 1 on the support of $g$. Then, with the notation $x \in \mathbb{R}^{v} \times \mathbb{R}^{v} x=\left\{x^{\alpha} ; \alpha=1 \ldots 2 v\right\}$, and $f^{\prime}$ 's of the form $f=x_{j}^{\alpha} h$, we obtain

$$
\int d \bar{\mu} \frac{\partial g}{\partial x_{j}^{\alpha}}=0 \quad j=1 \ldots n \quad \alpha=1 \ldots 2 v \text { for all } g \in \mathscr{C}_{\mathscr{O}}^{1} .
$$

Thus the derivations of $\bar{\mu}, \bar{\mu}$ considered as a distribution, vanish on the open connected set 0 . 
Therefore this distribution equals a constant function $C$ on $\mathcal{O}$ [15] and Property 2 follows.

3) With Equation (21) and Property 2 applied to each $D_{\alpha}^{\pi}$ we see that $\bar{\mu}$ is proportional to the Lebesgue measure on each $D_{\alpha}^{\pi}$. Moreover, the proportionality constant is independent of $\alpha$ and $\pi$ by the connectedness of $\mathscr{C}$, i.e.

$$
d \bar{\mu}=C d x_{1} \ldots d x_{n} \quad \text { on } \mathscr{C}
$$

and by the definition of $\bar{\mu}$ the Lemma 2 is thus proved.

Proof of Theorem 1. It follows from Lemma 2 (with $\Lambda$ replaced by $\left\{q \in \Lambda ; \chi_{d}(\{q\} \cup Y)\right.$ $=1\}$ ) and Lemma 1 that for any bounded open $\Lambda$ in $\mathbb{R}^{v}$, the conditional probabilities satisfy for almost all $Y$ in $\Omega_{\bar{A}^{c}}$

$$
\mu_{\bar{\Lambda}}\left[d X^{n} ; Y\right] \cong \chi_{d}(X \cup Y) e^{-\beta H_{\bar{A}, Y}\left(X^{n}\right)} C_{n, \bar{\Lambda}, Y}^{i} d x_{1} \ldots d x_{n}
$$

on each connected, open, component $\mathscr{C}^{i}$ of $\Omega_{\bar{\Lambda}, n}$.

To conclude the proof we must show that (23) holds, with $\bar{\Lambda}$ replaced by $\Lambda$, on all of $\Omega_{\Lambda, n}$. To do this we use the following lemma:

Lemma 3. Let $M_{\alpha}=\left\{q \in \mathbb{R}^{v} ;|q|<\alpha\right\}$ be the open $\alpha$-ball, $\alpha>0$. Then for any KMS-state $\mu$ of the infinite system,

$$
\int d \mu\left|X_{\partial M_{\alpha}}\right|=0
$$

for all but at most countably many $\alpha$.

Proof. The lemma follow easily from the fact that for a KMS state the expected number of particles in any finite region is finite.

From Lemma 3 it follows that there exists a bounded open set $M \supset \Lambda$ such that

i) $M$ is "the" set introduced in the definition of loose packing, and

ii) $\int d \mu\left|X_{\partial M}\right|=0$.

Consider Equation (23) with $\Lambda$ replaced by $M$. Using the compatibility condition Equation (8) (with $M$ replaced by $\bar{M}, \Lambda$ replaced by $\bar{\Lambda}$, and the expression Equation (23) for $\mu_{\bar{M}}[d X ; Y]$ ), it then follows in fact that for any loosely packed KMS-state and any bounded Borel set $\Lambda$ in $\mathbb{R}^{v}$

$$
\mu_{\Lambda}\left[d X^{n} ; Y\right] \cong \chi_{d}(X \cup Y) e^{-\beta H_{\Lambda, Y}\left(X^{n}\right)} C_{n, \Lambda, Y} \quad d x_{1} \ldots d x_{n}
$$

for almost all $Y$. This concludes the proof of Theorem 1.

Theorem 2. Any Canonical Gibbs State $\mu$ such that $\mathfrak{A} \subset \mathscr{L}^{1}[\mu], f \cdot\{g, H\} \in \mathscr{L}^{1}[\mu]$ for all $f, g \in \mathfrak{U}$, and in the case of hard core $\Phi\left(q_{1}, q_{2}\right) \rightarrow \infty$ for $\left|q_{1}-q_{2}\right| \rightarrow d$, is a KMS-state.

This converse statement follows immediately from Equation (10) by integration by parts which are justified by the conditions $\mathfrak{A} \subset \mathscr{L}^{1}[\mu]$ and the condition on the potential.

\section{Conclusion}

We have shown that any KMS-state is a canonical Gibbs state. In Ref. [12] it is proved under certain restrictive assumptions on the potential that any canonical Gibbs state is a mixture of Gibbs states with (possibly) different activities, i.e. 
extremal canonical Gibbs states are also extremal Gibbs states (the converse was also proven there). Since then, same result was established with considerably fewer restrictions on the potential. (It was shown by Goldstein, Aizenman, and Lebowitz [13] and by Preston [13] that a similar relation exists between (generalized) microcanonical Gibbs states and Gibbs states; their extremal points coincide). These results indicate again the equivalence between the different canonical ensembles (micro, macro, grand) commonly used for the description of macroscopic systems in equilibrium; it also shows the usefulness of the concept of classical KMS-states introduced in [7] and further developed in [11].

It is also possible to understand, in an intuitive way, that because the classical KMS-condition is derived as a consequence of stability of stationary states to (local) perturbations which conserve particle number, it leads to canonical (rather than "grand-canonical") Gibbs states. The situation in quantum systems with perturbations which conserve particle numbers has recently been investigated [16].

We also note here that the relationship between KMS-states and Gibbs states established here for continuous particle systems was established by van Hemmen [17] for harmonic crystals.

Finally, we should emphasize that insofar as it does not allow for coincident particles our definition of $\Omega$ (for $d=0$ ) is not the one used most frequently in statistical mechanics. In fact, for the usual definition [i.e., that $\Omega$ is the set of unordered sequences $\left\{\left(q_{i}, p_{i}\right)\right\}$, such that $\left.q_{i} \rightarrow \infty\right]$ it is not a consequence of the KMS condition that the local measures $\mu_{\Lambda}[d X ; Y]$ be absolutely continuous. Indeed, one can show, along the lines of the argument of this paper, that in this more general case a KMS state is, in effect, a canonical Gibbs state for a system of particles of different types--single particles, double particles ${ }^{4}$, triple particles, and so on. And, it may be shown, under assumptions similar to those of [12] or [13], that such a state is a "mixture" of Gibbs states (for the system of several types of particles). This result is what we should expect, since a) multiple particles cannot be separated by means of a Hamiltonian time evolution (or any other evolution for which the particles are intrinsically indistinguishable), therefore b) just like ordinary Gibbs states, Gibbs states for systems of multiple particles are stable (in the sense of [9]), and hence c) are KMS, since the KMS condition is a consequence of stability [9].

Note. This work was done independently by the authors in the United States and the authors in Lausanne.

\section{References}

1. Ruelle,D.: Statistical mechanics. New York: Benjamin 1969

2. Lanford,O.E., Ruelle,D.: Comm. math. Phys. 13, 194 (1969)

3. Ruelle,D.: Commun. math. Phys. 18, 127 (1970)

4. Dobrushin, R.L.: Theory Prob. Appl. 13, 197 (1968)

5. Gruber, C., Lebowitz,J.L.: Commun. math. Phys. 41, 11 (1975)

6. Haag, R., Hugenholtz, N., Winnink, M.: Commun. math. Phys. 5, 215 (1967)

7. Gallavotti,G., Verboven,E. Nuovo Cimento 28, 274 (1975)

8. Haag, R., Kastler, D., Pohlmeyer,E. : Commun. math. Phys. 38, 173 (1974)

9. Aizenman, M., Gallavotti,G., Goldstein, S., Lebowitz,J.L.: Commun. math. Phys. 48, 1 (1976)

4 Two particles with the same position and momentum 
10. Gurevich,B.M., Suhov,J.M.: Preprint Marseille 1975

11. Pulvirenti, M.: Stability, equilibrium, and KMS for infinite classical systems. Preprint 1976

12. Georgi,H.O.: Commun. math. Phys. 48, 31 (1976)

13. Goldstein,S., Aizenman,M., Lebowitz,J.L.: (to appear), and Preston,C. (to appear) have proven equivalence between general types of micro-Gibbs states (including canonical) and Gibbs states Georgi,H.O., have been able to relax some of the restrictive conditions on the potentials used in [12]

14. Lanford,O.E.: Dynamical systems theory and applications. Lecture Notes in Physics, Vol.38. Berlin-Heidelberg-New York: Springer 1975

15. Schwartz,L.: Theory of distributions. Paris: Hermann 1966

16. Araki,H., Haag, R., Kastler,D.: Preprint 1976

17. Van Hemmen, J.L.: Dynamics and ergodicity of the infinite harmonic crystal. Thesis, University of Groningen (1976)

Communicated by J. L. Lebowitz

Received January 3, 1977 